\title{
Leyomioma of the Gallbladder An Extremely Uncommon Diagnosis A Case Report
}

ISSN: 2637-7632

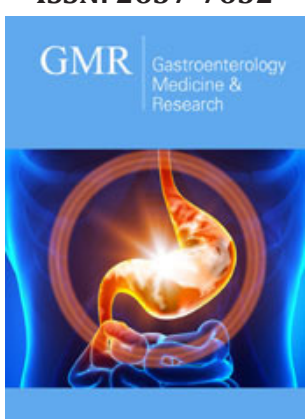

*Corresponding author: Carlos Enrique Luna-Guerrero, Department of General Surgery, Hospital General de Zona 5. IMSS. Sonora, Mexico

Submission: 海 June 18, 2020

Published: 此 December 09, 2021

Volume 6 - Issue 3

How to cite this article: Ivan Rodolfo Martínez-García, Luis Armando LópezRico, Carlos Enrique Luna-Guerrero, Edgar Vargas-Flores. Leyomioma of the Gallbladder An Extremely Uncommon Diagnosis A Case Report. Gastro Med Res. 6(3). GMR. 000636. 2021.

DOI: $10.31031 /$ GMR.2021.06.000636

Copyright@ Carlos Enrique LunaGuerrero, This article is distributed under the terms of the Creative Commons Attribution 4.0 International License, which permits unrestricted use and redistribution provided that the original author and source are credited.
Ivan Rodolfo Martínez-García, Luis Armando López-Rico, Carlos Enrique Luna-Guerrero* and Edgar Vargas-Flores

Department of General Surgery, Hospital General de Zona 5. IMSS. Sonora, Mexico

\section{Introduction}

Mesenchymal gallbladder neoplasms are sporadic; individually, very few gallbladder leiomyoma cases have been described, and all of them are diagnosed in patients with diseases of the immune system. A case report of a vesicular leiomyoma is described in a 41-year-old male patient without immune compromise. The patient lacked a significant past medical history and presented to the general surgery outpatient clinic with clinical data of acute cholecystitis and an ultrasound that reported cholelithiasis with no acute findings. It was sent to the emergency department for evaluation. Laboratory studies were performed, and the abdominal ultrasound was repeated, persistent symptoms were the primary indication to perform a laparoscopic cholecystectomy. He was discharged after clinical improvement after five days of hospital stay. Subsequent immunohistochemical study of the gallbladder specimen yielded the diagnosis of leiomyoma of the gallbladder fundus, with a positive SMA marker.

\section{Case Report}

41-year-old male patient, with no significant past medical history presented with intermittent right upper quadrant colic pain, occasionally accompanied by vomiting that spontaneously subsided attended an outpatient general surgery clinic with an abdominal ultrasound reporting non-acute cholelithiasis. It was decided to send him to the emergency department due to clinical exacerbation data. Laboratory studies reported no relevant data. The patient was scheduled for an emergent cholecystectomy (Figure 1). Transoperative findings were consistent with thickened gallbladder wall, firm adhesions to the momentum, and the liver. Puncture decompression of the gallbladder revealed purulent leakage material. Clinical deterioration with persistent tachycardia precluded an optimal postoperative evolution. A complete blood count listed a decrease in hemoglobin levels to $7 \mathrm{gr} / \mathrm{dL}$. An abdominal ultrasound reported free fluid in the parietocolic gutter and in the space of Morrison. The patient was scheduled for surgical exploration. 500cc of hemoperitoneum was found in the form of abundant clots, without active bleeding. Abdominal lavage with the placement of drains was performed. The evolution of the patient was uneventful and he was discharged on the fifth day of hospital stay. The subsequent pathology analysis and immunohistochemical study of the surgical piece yielded the diagnosis of a $1.7 \mathrm{~cm}$ background gallbladder leiomyoma, with a positive SMA marker, KI-67, CD-117, desmin, CD-34, and S-100 negative (Figure 2). 


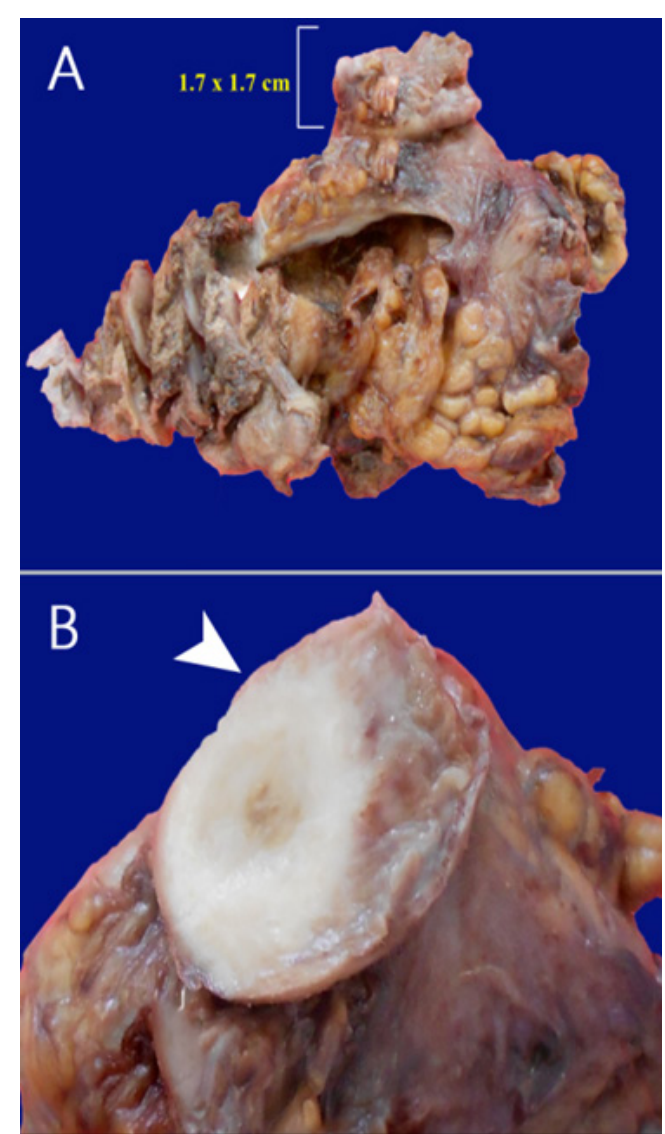

Figure 1:

(A). Gross pathology specimen of the gallbladder. A $1.7 \mathrm{~cm}$ diameter mass is found on the subserosal space. (B). Sharply circumscribed, round, firm, grayish white, "raw silk" and whorled cut surface (white arrowhead).

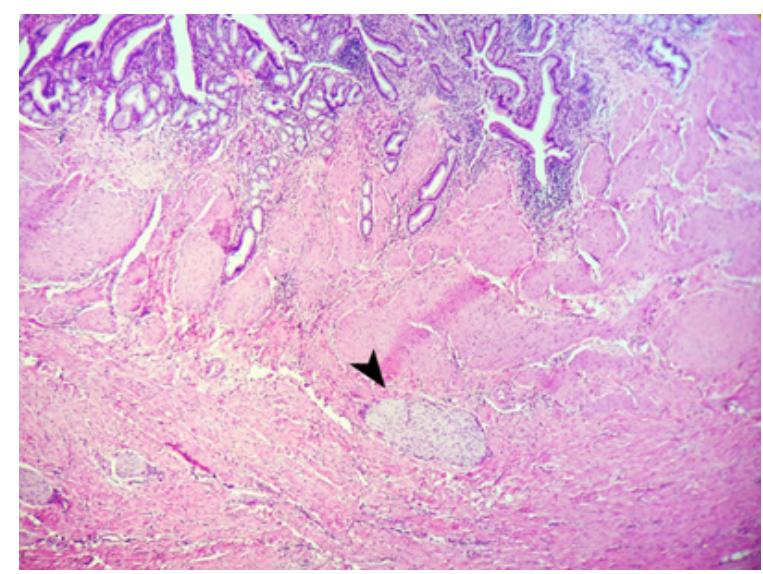

Figure 2: Whorled (fascicular) pattern of smooth muscle bundles separated by well vascularized connective tissue (black arrowhead). Elongated smooth muscle cells with eosinophilic or occasional fibrillar cytoplasm and distinct cell membranes.

\section{Discussion}

The leiomyomas are benign tumors of smooth muscle, commonly originate in the female genital tract (95\%) but can develop anywhere in the presence of smooth muscle fibers. Microscopic smooth muscle fibers with a fascicular pattern with eosinophilic cytoplasm and an elongated nucleus are observed microscopically. Immunohistochemically, the smooth muscle differentiation markers expressed are SMA, desmin, and h-Caldesmon, and are commonly negative for CD34, CD117, and S100 [1]. In a study carried out in Chile by Torres-Q [2] 5,699 biopsies were evaluated from 1998 to 2007, of which 4.9\% reported benign tumor lesions, the most frequent being gallbladder polyp (45.5\%) and an only one case of leiomyoma was found (0.4\%) [2]. So far, only one case of solitary vesicular leiomyoma has been described in the English literature in previous studies of benign gallbladder neoplasms, and only 3 cases in which a systemic leiomyomatosis in the context of infection by Epstein Barr virus-associated with immunodeficiency due to HIV, severe combined immunodeficiency and Wiskot-Aldrich syndrome [3]. The frequency of benign mesenchymal gallbladder tumors remains unknown. The incidence of gallbladder sarcomas is estimated to be around $2 \%$. The study of the tissue by biopsy (usually after performing a cholecystectomy) is necessary to establish the definitive diagnosis and thus exclude the possibility of leiomyosarcoma and other sarcomas. Therefore, leiomyoma should be taken into account in the differential diagnosis of gallbladder tumors, not only in immunosuppressed patients but also in patients with normal immune function [3].

\section{References}

1. Wachter DL, Buttner MJ, Grimm K, Hartmann A, Agaimy A (2010) Leiomyoma of the gallbladder: a case report with review of the literature and discussion of the differential diagnosis. Journal of Clinical Pathology 63(2): 177-179.

2. Torres GA (2009) Benign neoplasms of gallbladder, a national paradigm. Revista ANACEM 3: 2.

3. Segura-Sampedro JJ (2012) Gallbladder leiomyoma in absence of immune system disorders: An unusual diagnosis. Rev Esp Enferm Dig 104(7): 381-383. 